\title{
Le giroflier à Madagascar : essai d'histoire et de géographie coloniales, 1896-1958
}

Mathilde Cocoual et Pascal Danthu

\section{OpenEdition}

\section{Journals}

Édition électronique

URL : https://journals.openedition.org/geohist/1257

DOI : 10.4000/geohist. 1257

ISSN : 2264-2617

Éditeur

Association française de la Revue de géographie historique

\section{Référence électronique}

Mathilde Cocoual et Pascal Danthu, « Le giroflier à Madagascar : essai d'histoire et de géographie coloniales, 1896-1958 », Revue de géographie historique [En ligne], 12 | 2018, mis en ligne le 02 juin 2021, consulté le 12 juin 2021. URL : http://journals.openedition.org/geohist/1257 ; DOI : https:// doi.org/10.4000/geohist.1257

Ce document a été généré automatiquement le 12 juin 2021.

Ce(tte) œuvre est mise à disposition selon les termes de la Licence Creative Commons Attribution Pas d'Utilisation Commerciale - Pas de Modification 4.0 International. 


\title{
Le giroflier à Madagascar : essai d'histoire et de géographie coloniales, 1896-1958
}

\author{
Mathilde Cocoual et Pascal Danthu
}

\section{NOTE DE L'AUTEUR}

\section{Remerciements}

Cette étude a été réalisée dans le cadre d'un appui du Ministère des Affaires étrangères français (Projet FSP PARRUR). Les auteurs remercient Hervé Duchaufour pour son soutien, Stéphane Rey, Éric Penot et Michel Jahiel pour leurs conseils avertis, Bidy Benavory pour ses traductions et ses éclairages précieux et Serge Volper (Bibliothèque historique du Cirad) pour la fourniture des photographies.

1 «Longtemps la bande côtière de l'est [de Madagascar] a paru la région de colonisation par excellence $»^{\mathrm{i}}$. C'est en ces termes qu'Hildebert Isnard ${ }^{\mathrm{ii}}$, professeur de géographie des pays tropicaux à la faculté des Lettres d'Aix-en-Provence, introduit son étude de la colonisation agricole sur la côte orientale de Madagascar en 1951. Il y dresse à la fois le bilan et les perspectives qui s'offrent à la colonie française en termes d'exploitation agricole et y met en exergue la réussite de l'introduction de certaines espèces, parmi lesquelles le giroflier tient une place de premier ordre.

2 Le giroflier est un arbre de la famille des Myrtaceae, originaire des îles Moluques en Indonésie. Il produit un bouton floral communément appelé «clou de girofle», utilisé pour ses vertus culinaires et médicinales depuis l'Antiquité. Il est aussi à l'origine d'un second produit, l'essence de girofle obtenue par distillation des clous ou des feuilles (Maistre, 1964 ; Danthu et alli, 2014).

3 Subtilisé aux Hollandais sur ordre de Pierre Poivre - botaniste et Intendant des îles de France et de Bourbon (actuelles îles Maurice et de La Réunion) - le giroflier est 
implanté à l'île de la Réunion (1777), à Sainte-Marie (1821) puis à Madagascar et aux Comores.

4 Madagascar, Sainte-Marie et l'archipel des Comores deviennent progressivement des fournisseurs importants du marché mondial du clou de girofle, mais loin derrière les colonies anglaises de Pemba et de Zanzibar, au moins jusque dans les années 1960 (Martin, 1991).

5 Dès 1896, le gouvernorat de Madagascar, avec à sa tête Gallieni, impulse une politique de colonisation agricole qui transforme en profondeur la physionomie de la GrandeÎle ${ }^{i i i}$. Madagascar, devient dès lors, un laboratoire d'essai et de propagation des essences tropicales, principalement sur la côte est de l'île qui réunit les conditions écologiques et climatiques adéquates.

6 Cette transformation du paysage agricole malgache est due, tout autant à l'action des colons européens et bourbonnais, qu'à l'adoption par les Malgaches de ces nouvelles cultures : girofle mais également café, vanille, plantes à parfum, épices, etc. Soucieux de mettre au service de la métropole les produits de la colonie, les administrateurs soutiennent et accompagnent le développement de ces cultures de rente par différents moyens : appui aux agriculteurs, valorisation des produits du giroflier sur les marchés européens, législation à l'exportation.

7 Pour autant, les étapes d'implantation et d'expansion du giroflier à Madagascar à partir de 1896 comme le rôle concret joué par l'administration coloniale dans l'essor de cette filière ont été peu étudiés.

8 En effet, le rapatriement en France des archives coloniales et le peu de moyens accordés aux Archives nationales malgaches comme au Ministère de l'agriculture n'ont pas permis jusqu'alors d'archiver la documentation ni d'étudier de manière approfondie cette filière agricole qui est pourtant aujourd'hui le premier produit d'exportation de Madagascar (Danthu et alli, 2014).

D'un point de vue historique, seules quelques études isolées et très partielles permettent de saisir l'évolution de cette culture dans l'océan Indien (Ranohintsoa, 1978; Deleage, 1990 ; Tiollier, 2012 ; Volper, 2011).

10 L'objectif de cet article est double: d'une part, comprendre le contexte historique et géographique de l'implantation et de l'expansion du giroflier à Madagascar, plus particulièrement sur la côte est - moins bien étudiée que sa consœur saint-marienne (Ranohintsoa, 1978) - et, d'autre part, analyser le rôle joué par l'administration coloniale dans la promotion de cette culture. Pour mener à bien cette étude, deux corpus de sources ont été mobilisés : les parutions du Journal Officiel de Madagascar et Dépendances $(J O M D)^{\text {iv }}$, journal d'information et de législation consacré à la colonie, et les écrits des administrateurs ou ingénieurs coloniaux ${ }^{v}$. 
Document 1 : Giroflier âgé de cinq ans (Fénérive)

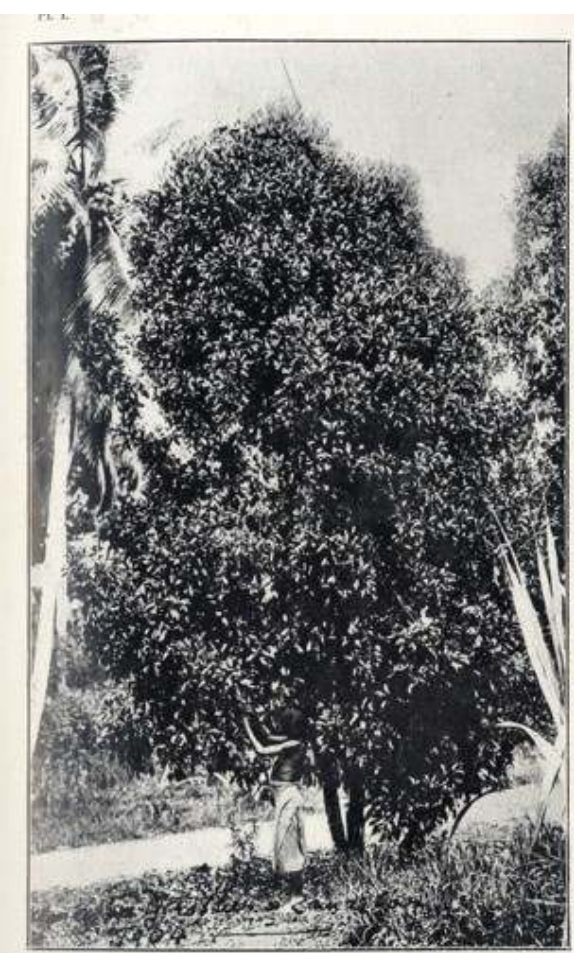

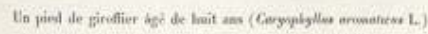

Source : Photographie prise par Ledreux ; Ledreux A., avril 1932, "Le cannelier à Madagascar", La Parfumerie Moderne, $n^{\circ}$ 4, pp. 179-187, p. 179. 


\section{Document 2 : Un jeune giroflier très probablement à l'Ivoloina (Tamatave)}

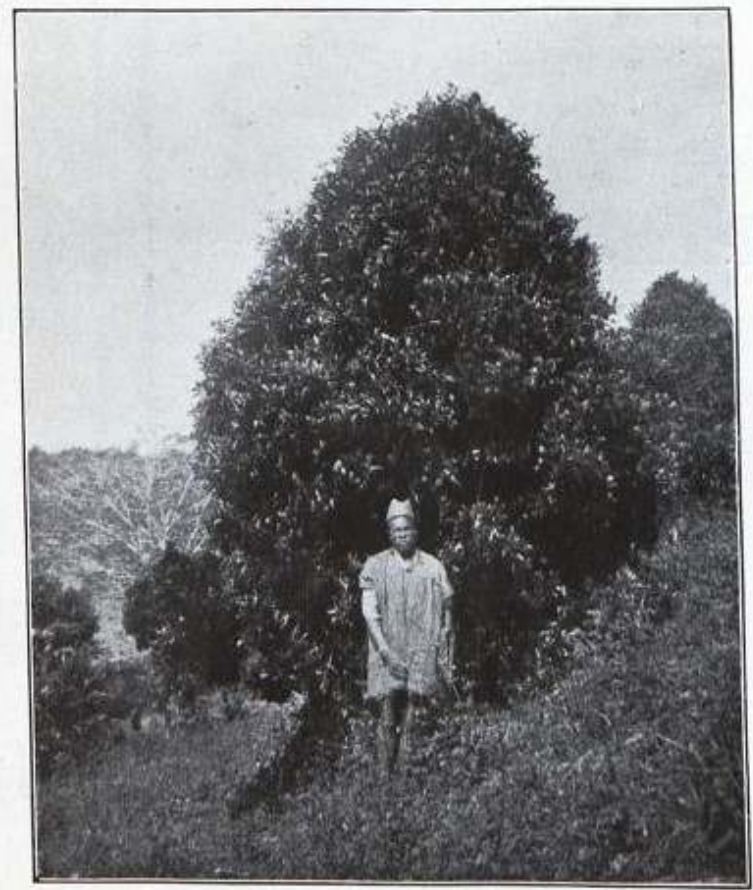

Giroflier agé de 5 ans (Fénérive) (Photo Ledreux)

Source : Photo d'Emile Prudhomme à qui Gallieni avait confié la création des Services agricole de la "Grande-Île". Arrivé à Madagascar en 1896, Prudhomme rentrera en France en 1905.Collection de la Bibliothèque Historique du Cirad (Nogent sur Marne) 
Document 3 : Photo d'Emile Prudhomme à qui Gallieni avait confié la création des Services agricole de la "Grande-Île". Arrivé à Madagascar en 1896, Prudhomme rentrera en France en 1905

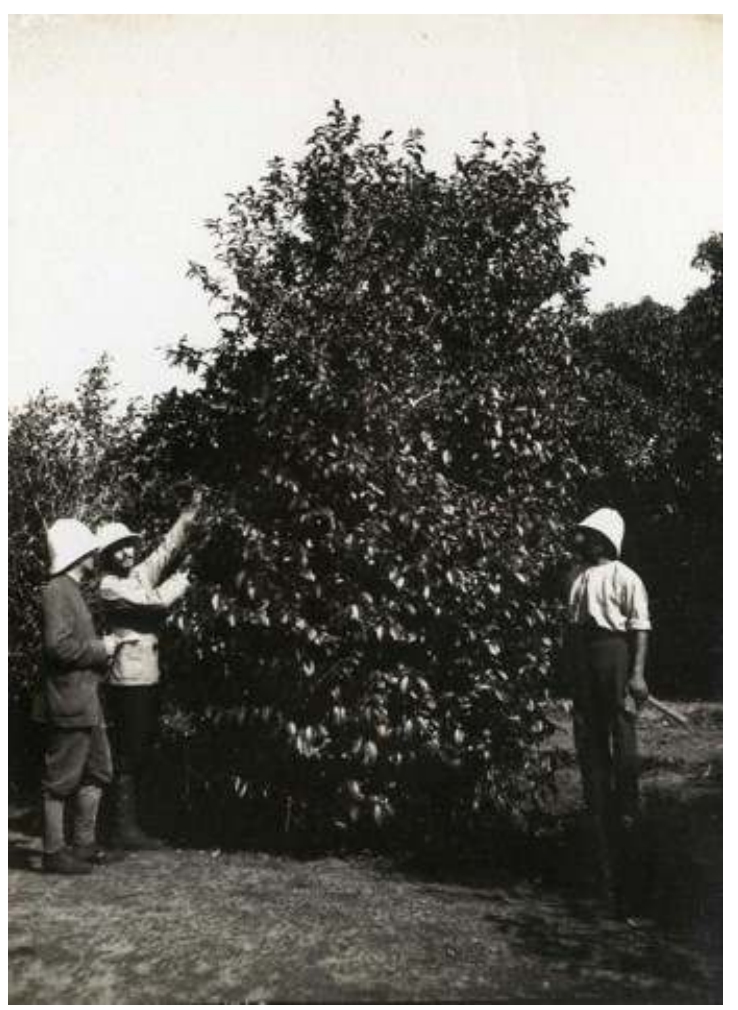

Source : Collection de la Bibliothèque Historique du Cirad (Nogent sur Marne)

\section{Expansion et culture du giroflier à Madagascar (1896-1958)}

\section{A. L'expansion du giroflier à Sainte-Marie et sur la côte est de Madagascar}

L'expansion du giroflier, comme celle des autres denrées coloniales introduites à Madagascar, est rapportée périodiquement par le JODM. Cette évolution agricole peut être suivie, de 1896 à 1903, par l'intermédiaire des «bulletins d'information des territoires civils $»^{v i}$ qui rapportent les différentes évolutions des nouveaux territoires administratifs et, de 1903 à 1920, au travers des immatriculations de domaines agricoles publiées au fil des attributions foncières concédées par l'Administration coloniale. À partir de ces données, il est dès lors possible d'appréhender les étapes d'introduction et d'expansion de cette essence.

En 1896, l'île Sainte-Marie est déjà couverte de girofliers vii alors que la côte est de Madagascar ne connaît que des tentatives d'introduction encore réduites. Le JODM fait cependant mention, en 1896, de plantations réussies à Vohémar et de débuts de culture à Mananjary. Il semblerait d'ailleurs que cette dernière localité soit le foyer initial de la culture du giroflier sur la Grande-Île. En effet, un bulletin officiel de la provinceviii y annonce, en 1897, la récolte de clous de girofle, ce qui suppose que les arbres étaient alors âgés d'au moins une dizaine d'années. Cette même année, des Malgaches commencent une plantation dans la baie de Tintingue, près de Manompana ${ }^{i x}$. À partir 
de 1898, l'Administration coloniale s'empare du contrôle des attributions de domaines.

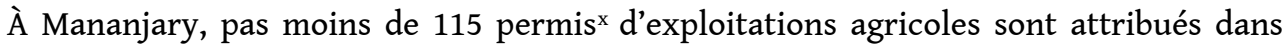
lesquels le girofle occupe une place prédominante. Le JODM est alors utilisé pour vanter les possibilités de colonisation agricole offertes par la côte orientale, notamment la province de Fénérive : «Ce sont des terrains fertiles, si l'on en juge par la vigueur et la belle venue des plantations indigènes qui entourent les villages; mais aucune expérience n'a encore été tentée pour ce qui est des cultures tropicales: café, cacao, vanille, girofle, etc. Cependant, les planteurs trouveront d'utiles indications sur ces cultures à l'île Sainte-Marie, où il existe des plantations de vanille, de café et surtout de girofle, qui constituent pour le pays une source de revenu $»^{x i}$. On peut cependant noter que ni Galliéni ${ }^{x i i}$, ni Prudhomme ${ }^{x i i i}$ ne semble accorder un grand avenir au girofle par rapport à la vanille ou au caoutchouc ${ }^{\text {xiv }}$ (Danthu et alli, 2016).

Document 4 : Distillerie indigène de feuilles de giroflier, Alambic Deroy (Mananara)

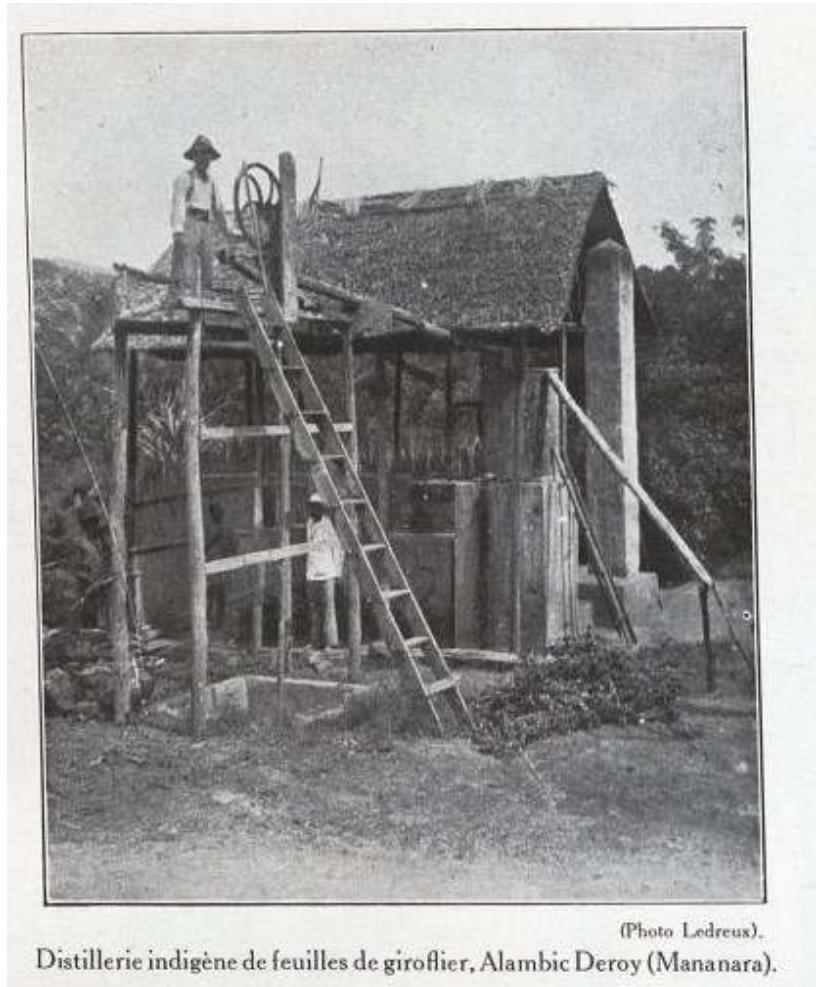

Source : photographie prise par Ledreux Ledreux A., avril 1932, "Le cannelier à Madagascar",La Parfumerie Moderne, n 4, pp. 179-187, p. 185.

13 Les grandes entreprises coloniales investissent de manière croissante dans ces denrées coloniales. La Compagnie Marseillaise obtient, en 1899, une concession de 100 hectares à Fénérive en vue de cultiver café, girofle, vanille, etc ${ }^{\mathrm{xv}}$. Entre 1900 et 1903, ce sont successivement les territoires de Mananara, Tamatave, Andovoranto puis Mahanoro qui accueillent la culture du giroflier (carte 1).

14 Si les concessions se multiplient sur la côte est, Sainte Marie se spécialise peu à peu dans la «monoculture » du giroflier, ce dernier recouvrant une large superficie de lîle. En 1899, un bulletin du JODM rapporte que dans cette île, " 63 indigènes s'adonnent à l'agriculture coloniale sur une superficie de 781 hectares dont principalement du girofle auxquels 
ils convient d'ajouter les domaines européens dont 7 concessions de plus de 100 hectares et 8 concessions de moins de 100 hectares $»^{\mathrm{xvi}}$.

À partir de 1903, apparaissent dans le JODM les premières immatriculations de propriétés indiquant le nom du planteur, le titre de la propriété, la localité, la superficie concernée et les essences cultivées. Entre 1903 et 1920, 43 immatriculations de domaines agricoles produisant du girofle à Sainte-Marie et sur la côte est de la Grande-île sont mentionnées (cartes 2 et 3 ). Sur ces 43 enregistrements, 15 immatriculations concernent Sainte-Marie et 28 la côte est de Madagascar en particulier dans la proximité de Fénérive. Compte tenu de la taille respective des deux régions, cette répartition confirme les travaux de Rahonintsoa (1978) qui postulent que les Saint-Mariens ont adopté de manière plus large que sur la Grande-île le système d'immatriculation ${ }^{\text {xvi }}$ afin de conserver leur droit sur la terre.

\section{Document 5 : Distillerie à Sainte-Marie}

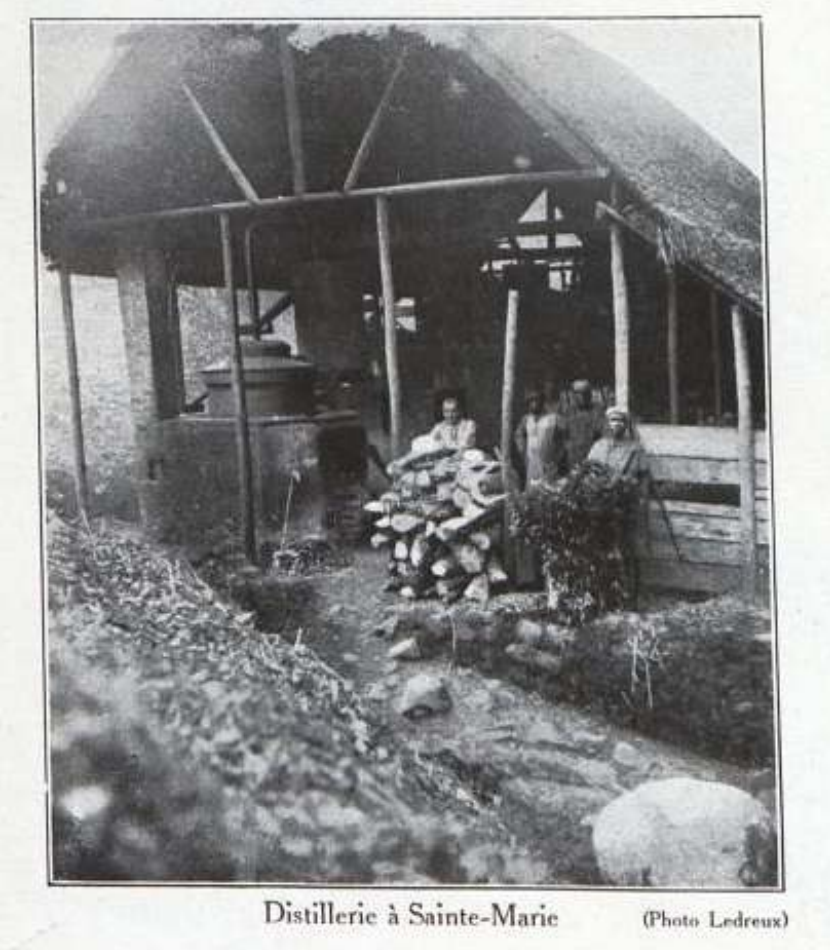

Source : Photographie prise par Ledreux A., avril 1932, "Le cannelier à Madagascar", La Parfumerie Moderne, $n^{\circ}$ 4, pp. 179-187, p. 185.

Ces immatriculations obéissent à des vagues d'enregistrements comme c'est le cas en 1913 ou en $1917^{\text {xviii }}$. La première vague est liée à la multiplication des ateliers de distillation (dont les premiers sont attestés dès 1906 ou 1911 ${ }^{\text {xix})}$ et à l'agrandissement des plantations. La seconde semble être induite par la réorganisation du service de l'Agriculture en 1916 (Volper, 2011) dont les administrateurs sont désormais plus nombreux et mieux formés. Pour autant, ce tableau n'est pas exhaustif compte tenu du fait que les immatriculations publiées ne couvrent pas l'ensemble des domaines exploités puisqu'elles ne concernent pas, selon l'article premier de la loi du 9 mars 1896, "les parcelles sur lesquelles les habitants ont déjà bâti et celles qu'ils ont l'habitude de cultiver » (Rahonintsoa, 1978). 
17 Les immatriculations de domaines agricoles couvrent ainsi, en 1930, environ 130 hectares pour Sainte-Marie où le girofle est prédominant et environ 1700 hectares $^{\mathrm{xx}}$ pour la côte est de la Grande-île, où le giroflier, d'abord secondaire face au café ou à la vanille, prend une place de plus en plus importante (carte 4).

Ces chiffres, établis uniquement à partir des immatriculations de domaines du JODM, sont sans aucun doute sous-estimés par rapport à la réalité. En effet, l'étude de Rahonintsoa (1978) rapporte que 820 hectares sont mis en valeur par des girofliers à Sainte-Marie dans les années 1930.

Ces données permettent d'écarter la vision d'une extension en tâche d'huile partant de Sainte-Marie vers la côte est de Madagascar puis s'étendant progressivement de la baie de Tintingue vers le nord et le sud. Il semble que l'extension résulte plutôt d'une implantation en mosaïque, colonisant par à coup des territoires parfois très éloignés, dépendant de facteurs à la fois climatiques, humains, matériels et financiers, sans schéma directeur préétabli.

Document 6 : Planche botanique extraite de Ledreux A. 1932, Le Giroflier à Sainte-Marie et Madagascar.

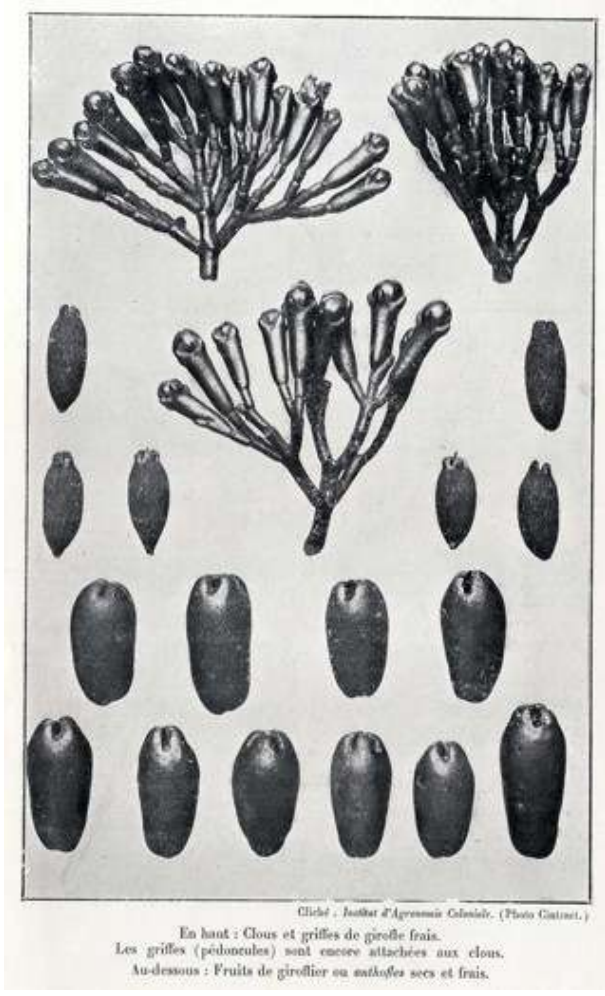

Source : Ministère des Colonies. Institut National d’Agronomie Coloniale. Nouvelle Série n 161.

L'analyse des immatriculations comme des bulletins des territoires civils révèle une grande disparité dans la superficie des domaines. À côté des grandes concessions européennes, de 100 à 800 hectares, la majorité des domaines - appartenant à des petits colons européens ou créoles et à des Malgaches - oscille entre 10 ares et 11 hectares.

À partir des années 1920, le JODM ne signale plus aucune immatriculation de propriété produisant du girofle, il se focalise désormais sur l'encadrement de la distillation des essences de girofle. 
Document 7 : Timbre-poste émis en 1946 dans une série illustrant les principales productions agricoles de Madagascar

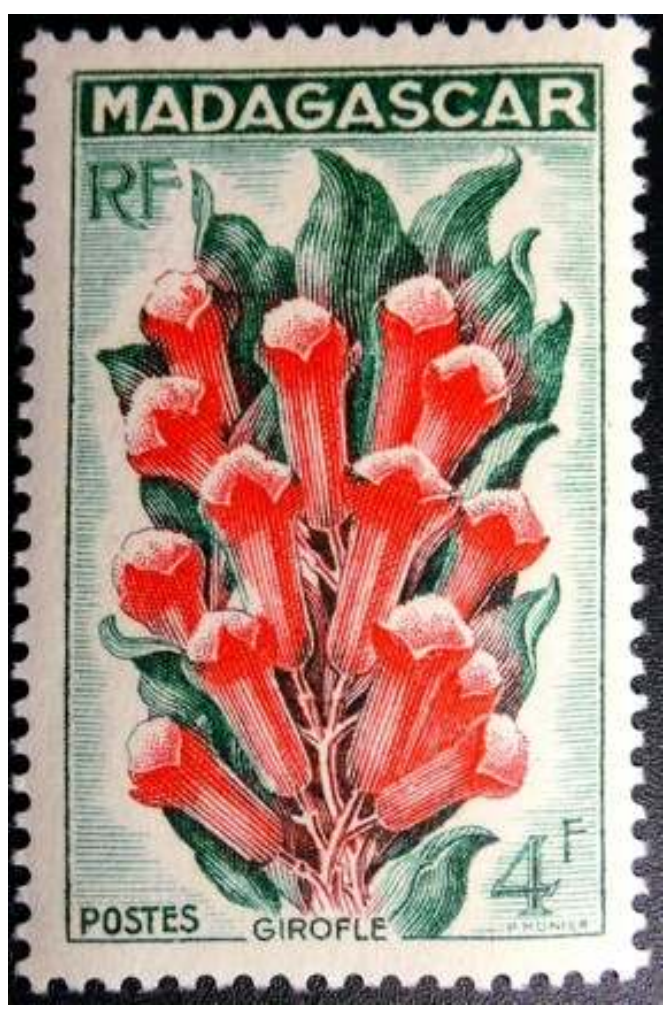

\section{La production d'essence de girofle}

Les premières tentatives de distillation de feuilles et griffes (pédoncule floral séparé du clou après cueillette) de girofle ont lieu à partir de 1906 (Rahonintsoa, 1978). Elles permettent d'obtenir une essence riche en eugénol, employée en industrie, en parfumerie, en cosmétique, dans la composition d'arôme alimentaire ou en pharmacologie. Chronologiquement, son premier usage est celui de plastifiant dans la fabrication des vernis servant à peindre les cocardes des ailes d'avions ${ }^{x \times i}$. L'eugénol se révèle aussi être un antiseptique puissant utilisé dans les pathologies dentaires (Duclos, 2012 ; Danthu et alli, 2014). C'est enfin la principale molécule de base pour l'hémisynthèse de la vanilline d'après le protocole que Georges de Laire a mis au point en $1876^{\text {xxii }}$ (Zolesio, 2016). D’abord réservée aux industriels de la métropole qui distillaient les clous importés en France, la distillation du girofle, se développe sur le territoire de la colonie au début $\mathrm{du} \mathrm{XX}^{\mathrm{e}}$ siècle et prend un essor grandissant durant la Première Guerre mondiale.

En 1902, un colon de Sainte-Marie, Plaire, de la plantation d'Ankarena située à la pointe méridionale de l'îlexiii, installe un alambic pour distiller les griffes normalement rejetées par les exploitants et qui assurerait, au regard du prix dérisoire de la matière première, un bénéfice important ${ }^{x x i v}$. À cette époque, il écarte la possibilité de distiller les clous, trop onéreux, mais il étudie l'éventualité de distiller les feuilles de girofliers. Selon Ranohintsoa (1978), il importe, en 1906, depuis la métropole, un alambic en cuivre, de marque Deroy Fils Ainé - fabricant parisien d'appareil de distillation. La réussite de l'entreprise incite les autres agriculteurs à suivre son exemple. Mais ce type 
d'installation nécessite un capital initial important ce qui explique la primauté des colons dans l'implantation des premiers ateliers de distillation. En 1911, les premières exportations d'essences de girofle sont acheminées à destination de la métropole pour un poids de 872 kilogrammes ${ }^{\mathrm{xxv}}$. La généralisation de la distillation, notamment auprès des producteurs malgaches, est impulsée par le colon Taybaly qui, en 1926, rachète l'épave du Cavalaire (rebaptisé Ivolina), cargo qui s'était échoué sur les récifs de l'île au Nattes, dont il utilise la tôle et la chaudière, toujours fonctionnelle, pour fabriquer un alambic artisanal. Le succès de cette construction l'encourage à renouveler l'expérience: des alambics de fabrication locale sont disséminés un peu partout dans les concessions et loués aux planteurs locaux. Les installations de Sainte-Marie occupent principalement l'ouest de l'île, à proximité des zones de production et des cours d'eau. Les JODM de 1926 font ainsi état de trois enquêtes de Commodo et Incommodo concernant la demande de concession des eaux des ruisseaux pour alimenter des distilleries à Sahavamba ${ }^{\text {xxvi }}$, Ambohitra ${ }^{x x v i i}$ et Antsarahakaxxviii. En 1931, 55 distilleries fonctionnent ainsi dans l'île (Rahonintsoa, 1978).

Qu'en est-il pour la côte est de Madagascar ? La première enquête de Commodo et Incommodo date du 10 novembre 1923, dans le district de Tamatave : «M. Oscar Pothin, colon, demeurant sur l'Ivoloina, canton de Vohidrotra, déclare vouloir installer un établissement pour distiller des huiles essentielles: girofle, cannelle, ylang-ylang, etc. "xxix. À partir de cette date et jusqu'en 1955, 44 unités de distillation font l'objet d'une enquête ou d'une autorisation de la part de l'Administration coloniale. D'abord réservées aux colons européens, les autorisations s'étendent ensuite aux Malgaches, principalement à partir de 1945. En premier lieu individuelles, ces autorisations sont ensuite délivrées par listes de personnes d'un même district. À côté des distilleries coloniales, pouvant posséder plusieurs alambics importés de métropole, des unités intermédiaires et des alambics de fortune se partagent la distillation des feuilles, griffes et parfois clous de girofle. Proche des ruisseaux et rivières pour approvisionner l'alambic en eau, ces postes de distillation sont majoritairement implantés à Soanierana, Ampasibe, Ampasina, Vatomandry et Fénérive. Seule une unité est déclarée durant toute la période à Tamatave, Mahanoro ou Manakara. L'implantation de ces unités n'est pas sans conséquence environnementales: le bois nécessaire à la distillation est recherché toujours plus profondément dans les forêts entraînant une déforestation signalée dès 1955 par Maistre ${ }^{\mathrm{xxx}}$. Cette question est aujourd'hui encore très prégnante (Danthu et alli, 2014).

Tout comme pour l'immatriculation des domaines, il est certain que le nombre d'alambics et de distillateurs est bien supérieur à l'effectif déclaré au sein du JODM. Si l'administration tente par la sanction d'obliger les propriétaires d'alambics à déclarer leurs appareils, ces mesures ne semblent pas avoir eu l'effet escomptéxxxi. Le planteur, qui se livre également à la distillation de cannes à sucre pour la confection de rhum, omet bien souvent de déclarer son alambic au chef de canton! 
Document 8 : Implantation des cultures de giroflier à Madagascar et dépendances entre 1898 et 1905

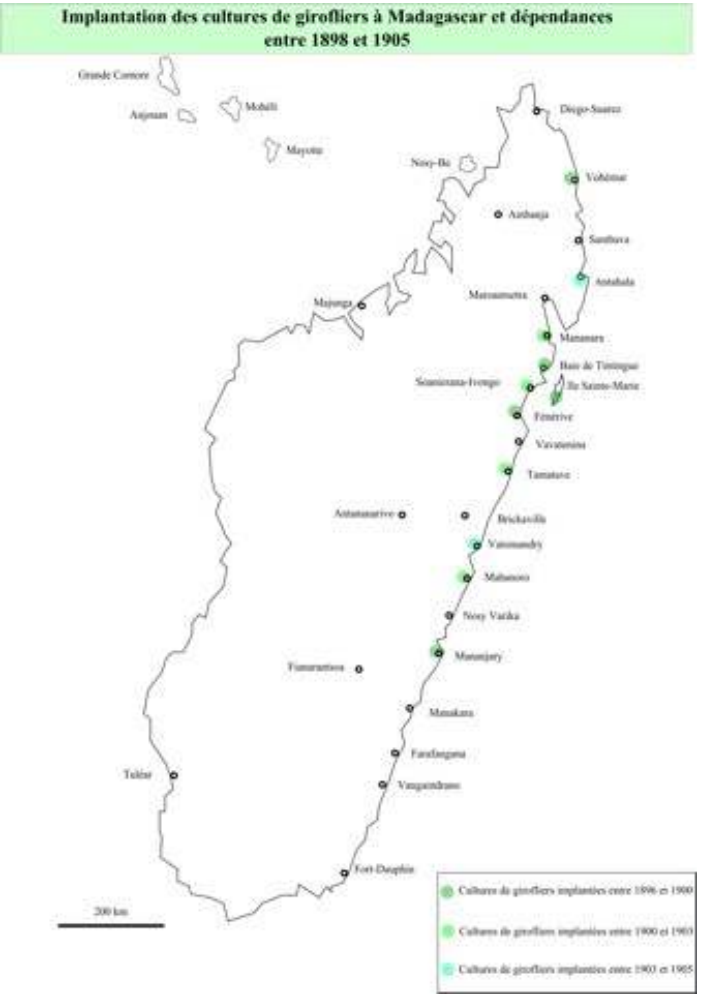

\section{B. La question de la main d'œuvre}

La récolte des clous nécessite de nombreux bras pour une courte période (de mioctobre à début décembre). Aujourd'hui encore cette question de la disponibilité d'une main d'œuvre abondante est un problème et une limite forte à la collecte des clous. Cette pénurie occasionne, à bien des reprises, la récolte d'une partie seulement des clous arrivés à maturité. Ce problème est déjà mis en exergue par l'Administration coloniale en 1897 qui déplore que la récolte de 1896 n'atteigne que 70 tonnes alors que la production potentielle est estimée à plus de 100 tonnes ${ }^{\text {xxxii. }}$. 


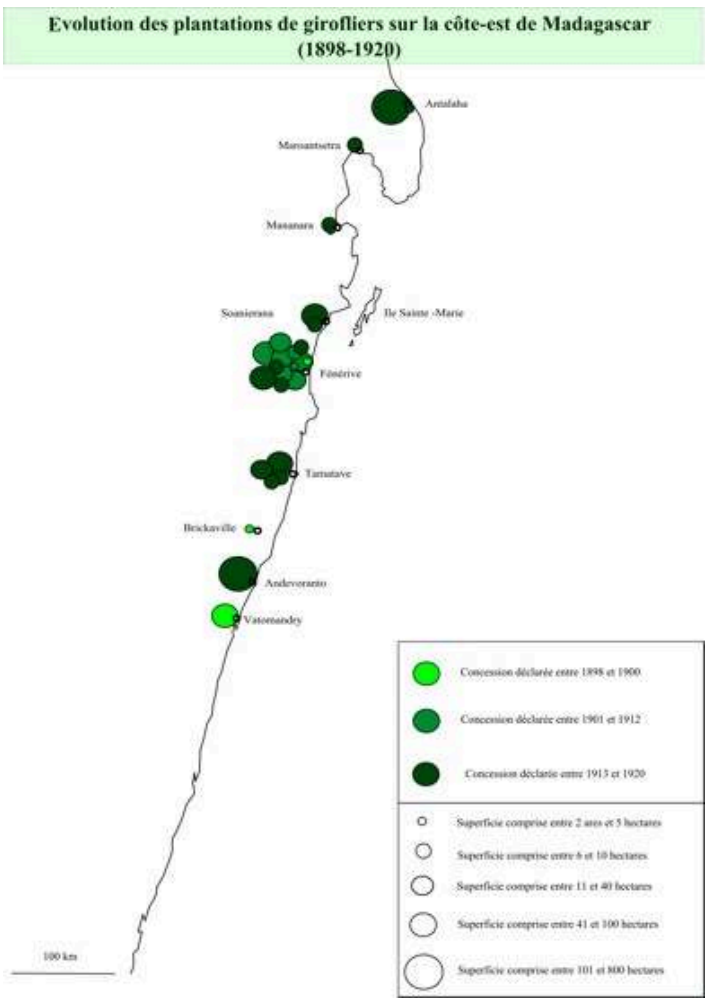

À Sainte-Marie, la récolte étant souvent une entreprise familiale, elle implique de ce fait les enfants. Aussi, des mesures sont prises pour rendre ces derniers le plus disponible possible durant la période clé. En 1898, un article du JODM consacré à l'enseignement à Sainte-Marie révèle que : «Les vacances ont dû être prolongées par suite de la cueillette des girofles qui est uniquement effectuée par les enfants; l'ouverture des vacances avait été pour cette raison reportée à l'époque de cette récolte " ${ }^{x x i i i} . \mathrm{Si}$, par la suite, le travail des enfants est combattu par le gouvernement, le législateur laisse planer un doute sur la question de leur emploi dans les travaux de cueillette, assimilés à un apprentissage. Ainsi, le 26 décembre 1936, le Gouverneur général Léon Cayla s'attarde sur cette question sans toutefois préconiser des sanctions contre le travail infantile : "Mon attention a été particulièrement attirée sur la question du travail de l'enfance. [...]. L'article 8 du décret du 22 septembre 1925 semble vouloir limiter le travail des mineurs aux simples travaux de champs (fécondation de la vanille, cueillette des clous de girofle, etc.). Ce texte appliqué strictement rendrait l'article 27 du décret du 14 janvier 1936 sans objet et supprimerait totalement l'apprentissage. Cela n'a certainement pas été dans l'intention du législateur. Il faudra donc que les chefs de district tiennent compte de cette interprétation, afin que les enfants ne soient pas empêchés de commencer à apprendre un métier avant l'âge de 18 ans »xxiv. 
Document 10 : Evolution des plantations de girfliers à l'île Sainte-Marie de Madagascar (1898-1920)

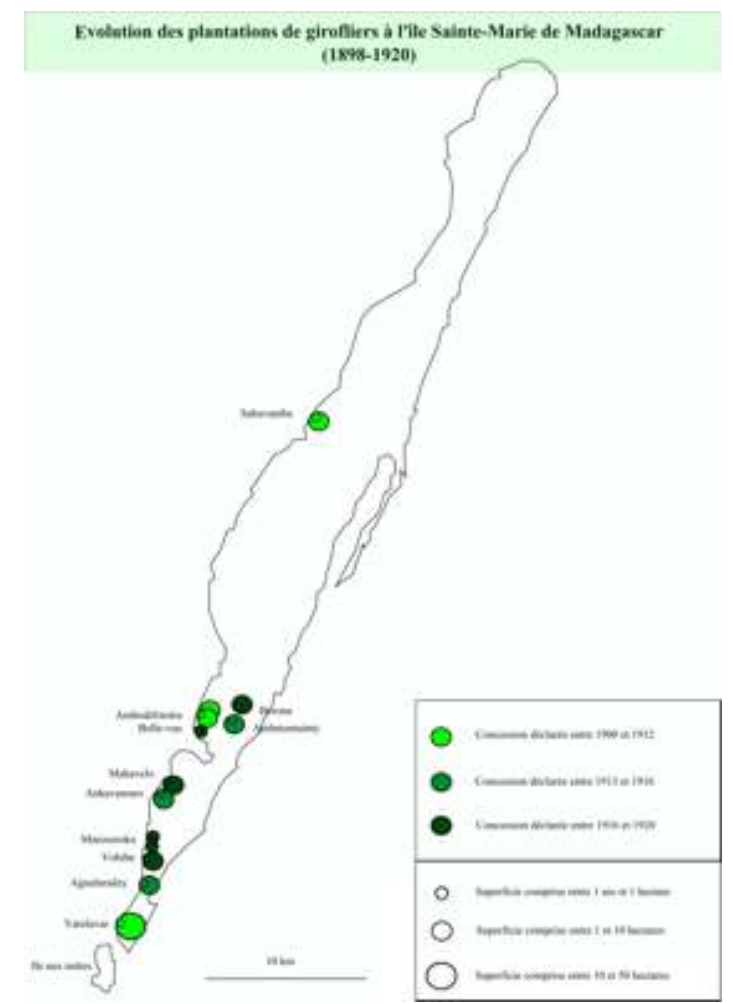

Pour faire face à la pénurie de main d'œuvre, il est fait appel à la population carcérale (Deleage, 1990). Ce recours, dans le cas du girofle, est spécifique à Sainte-Marie qui peut bénéficier de la disponibilité des prisonniers de la Maison de force de Sainte-Marie. On ne le rencontre pas sur la côte est de Madagascar. Cette mobilisation de la population carcérale se retrouve pour d'autres cultures comme celle de l'ylang-ylang à Nosy-Be ${ }^{\mathrm{xxxv}}$. Ainsi, les forçats, régulièrement mobilisés, deviennent des cueilleurs de clous. Cependant, le peu de soin accordé aux arbres, dont les branches sont régulièrement cassées, constitue l'inconvénient principal du recours aux prisonniers. Toutefois, cet appoint de main d'œuvre reste précieux. D'ailleurs, en 1921, l'Administration rend publique leur affectation à la fabrication d'essence. "Par décision provinciale du 27 septembre 1921, approuvée le 18 octobre 1921 par le Gouverneur général, la ration forte telle qu'elle est fixée par l'article 65 de l'arrêté du 27 juillet 1921 est allouée aux détenus employés, dans la province de Sainte-Marie, à la préparation de l'essence de girofle dans les usines des particuliers ${ }^{\text {xxvi }}$. Un prisonnier travaillant une journée touche 1,50 francs contre en moyenne 2,50 pour un ouvrier salarié.

Lorsque la récolte s'avère importante, les planteurs se plient au système du sambiamanana, coutumier sur la côte est. Le sambiamanana ou cueillette à mi-fruit repose sur le partage moitié-moitié des clous cueillis entre le propriétaire du giroflier et le cueilleur. Apparut en 1936 (Fremigacci, 2014), ce système est cependant « très vite vu par les colons aussi immoral que dangereux pour le pays, une pratique néfaste, démoralisante, aussi bien pour la main d'œuvre qu'elle habitue à des salaires injustifiés, que pour l'employeur qu'elle conduit à la ruine » (Fremigacci, 2014).

Si l'Administration coloniale n'a pu apporter que des réponses partielles aux besoins grandissants de bras pour la culture du giroflier, devenue une industrie saisonnière sur 
la côte est, elle a néanmoins obtenu des résultats probants dans l'encadrement de la filière à la production comme à l'exportation.

Document 11 : Implantation des cultures de Girofliers à Madagascar et dépendances en 1947

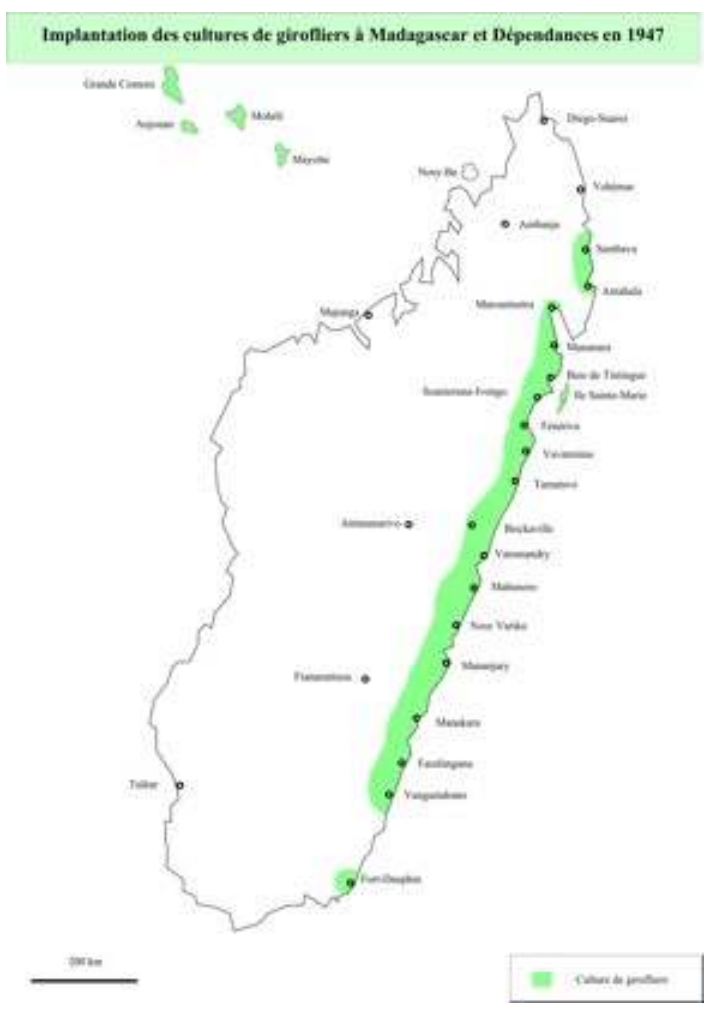

\section{L'action coloniale en faveur de la filière girofle}

\section{A. Favoriser l'extension des plantations et la diffusion des savoirs sur la culture du giroflier}

Selon Edmond François ${ }^{x x x v i}$, à partir des années 1920, l'Administration soutient l'adoption et la culture du giroflier par les petits agriculteurs malgaches. Il cite notamment l'action de l'administrateur Ballot « qui s'était tout spécialement attaché à la propagation des cultures indigènes "xxxviii. Il estime que, dans le seul district de Mananara, les Malgaches ont planté un million de girofliers de 1924 à 1926. Pour encourager la qualité de cette production, l'Administration récompense, lors de foires régionales, comme à Maroantsetra les 14 et 15 octobre 1933, les plus beaux clous présentés ${ }^{\mathrm{xxxix}}$. Les enseignants prennent aussi part à cette action. Par exemple, à partir de 1956-57, dans un village proche de Fénérive, un enseignant de l'école catholique, Jean Ratison, impose aux élèves de primaire de planter et de s'occuper de 30 à 40 arbres chacun ${ }^{x l}$. Au sortir de l'école, c'est donc tout naturellement que les élèves deviennent producteurs de clous de girofle, en récoltant les arbres qu'ils ont planté durant leur scolarité. Ces mesures s'inscrivent dans le prolongement d'expériences déjà couronnées de succès. Dès 1916, l'école régionale de Maroantsetra enseigne aux enfants à cultiver un jardin scolaire où le giroflier est prédominant. (Deleigne, 2010). 
32 Afin de fournir le matériel végétal nécessaire à cette expansion, la station d'essai d'Ivoloina, proche de Tamatave organise la multiplication en masse des girofliers au sein de ses pépinières. Les premiers travaux commencent en 1897 et les pépinières, réorganisées en 1902, permettent d'approvisionner tous les colons : « aussi bien ceux qui

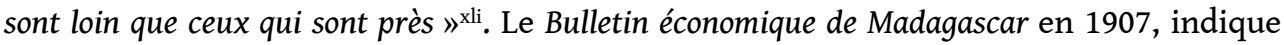
que la station d'Ivoloina prépare "de nombreux plants, de fortes demandes ayant été faites ${ }^{x \text { liii }}$. Le règlement fixe le prix d'un plant de giroflier en motte à cinq centimes et indique qu'il ne pourra être livré annuellement plus de 1000 pieds au même colon. Cette précision renforce l'idée d'une expansion importante de cette culture sur l'ensemble de la côte est.

\section{B. Encadrement et contrôle de la commercialisation des produits du giroflier}

33 En aval de la production, l'Administration coloniale encadre par voie législative les conditions de transport, de stockage et d'exportation des produits.

34 La première mesure prise, en 1897, en faveur des clous de girofle saint-mariens aux dépends des clous provenant de Zanzibar consiste en une taxation douanière favorable de 104 francs pour 100 kilogrammes contre 208 pour ceux provenant de Zanzibar xliii. En 1901 , ces droits de douanes sont réévalués, mais le différentiel est conservéxliv. Ces tarifs préférentiels sont vivement soutenus par la chambre d'agriculture de Sainte-Marie qui se fait le porte-parole des planteurs et colons ${ }^{\mathrm{xlv}}$.

L'Administration tente aussi d'améliorer les conditions de stockage des denrées coloniales au départ de Sainte-Marie et de la Grande-île: création et entretien d'entrepôts, surveillance des stocks, etc. Ces mesures qui entrent en vigueur à partir des années 1920 ont aussi pour finalité de mieux contrôler les quantités exportées et de prélever un certain nombre de taxes. Ainsi, à Sainte-Marie, un arrêté publié dans le JODM le $1^{\mathrm{e}}$ juillet 1922 instaure un droit de stationnement des marchandises sur les quais de la ville d'Ambodifototra et de l'îlot Madame ${ }^{x l v i}$ qui favorise encore les exportateurs de girofle : le raphia ou la vanille sont imposés de 0,10 francs par jour et par mètre carré occupé, le girofle est quant à lui soumis à une taxe inférieure de moitié.

Enfin, l'Administration coloniale promulgue des mesures visant à réduire les fraudes. Les premiers cas attestés d'adultération sont contemporains des premières exportations et émanent, non pas du territoire malgache mais des clients métropolitains. Ainsi, un article du JODM du 27 avril $1897^{\text {xlvii }}$ dénonce une fraude à la distillation ayant des conséquences néfastes pour la colonie : des distillateurs français remettent sur le marché les clous ayant été distillés alors qu'ils doivent être normalement détruits. Cette fraude a pour effet de grossir le volume de clous en circulation sur le marché et ainsi d'abaisser les cours de la matière première. L'Administration adopte un décret le $1^{\mathrm{er}}$ octobre $1898^{\mathrm{xlviii}}$ réglementant plus étroitement les conditions d'admission. En métropole, les clous destinés à la distillation seront désormais sous le contrôle des douanes qui s'assureront de leur destruction après distillation.

Dans la même optique, le gouvernement tente de limiter les fraudes sur les stocks exportés depuis Madagascar, notamment l'incorporation volontaire ou par négligence de déchets (feuilles, tiges, graviers, fientes de poules) ou la mise sur le marché de produits mal séchés, ce qui alourdit les lots et en péjore la qualité. Cette fraude est 
courante et elle a été largement utilisée dans d'autres filières malgaches comme celle du caoutchouc (Danthu et alli, 2016). Un arrêté du 15 novembre 1930xlix précise les normes de qualité de certains produits naturels de Madagascar et dépendances dont les clous de girofle pour lesquels une catégorisation est mise en place. Deux types de lots sont alors définis: le type $\mathrm{n}^{\circ} 1$, de qualité supérieure et le type "tout venant". Pour chacune de ces catégories l'arrêté définit le pourcentage acceptable de déchets ainsi que le taux d'humidité maximal admis.

De même, l'Administration tente d'éviter les vols par la mise en place de patente et de permis de circulation'. À partir de 1922, les transporteurs d'essences doivent justifier de la quantité et de la provenance de leurs huiles essentielles. L'absence de ces justificatifs implique une saisie immédiate de la marchandise qui est mise aux enchères avant de retourner dans le cycle de commercialisation.

L'ensemble de ces mesures a permis de réguler la qualité des clous et de l'essence de girofle provenant de la colonie et de ses dépendances. À partir de 1957, le gouvernement colonial crée la caisse de stabilisation des prix du girofle ${ }^{\text {li }}$. Elle a pour objectif de maintenir un prix d'achat fixe des produits, protégeant le producteur des effets néfastes de la fluctuation des cours et de la spéculation des exportateurs ${ }^{\text {lii. }}$.

Cependant, si le commerce des produits du girofle a connu, à partir de la seconde moitié du XIXe siècle, une croissance continue, l'intérêt pour ces marchandises en métropole n'allait pas de soi. Le gouvernement français a dû redoubler d'effort pour valoriser ces denrées auprès du grand public et trouver des débouchés nouveaux capables d'absorber l'augmentation croissante de la production.

\section{Valorisation des produits du giroflier malgache et recherche de débouchés}

Entre 1899 et 1920, le gouvernement colonial met à profit les différentes expositions internationales et nationales pour porter à la connaissance du public les denrées produites dans les colonies. Dans ces expositions, le girofle de Madagascar tient une place modeste mais qui mérite d'être évoquée. Pour permettre cette valorisation, les administrateurs provinciaux travaillent de concert avec les agriculteurs. Ainsi, le 16 mars 1899, le JODM publie ses recommandations destinées aux planteurs de SainteMarie afin de constituer les collections pour l'Exposition universelle de $1900^{\text {liii. En }}$ octobre 1901, les clous de girofle de Madagascar, quatrième produit agricole valorisé après le café, le cacao et la vanille, sont exposés au Concours général de Paris et aux

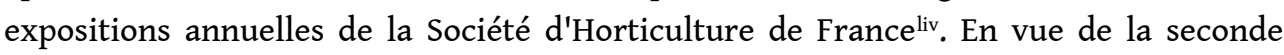
édition du Concours général de Paris, prévu en 1903, un article du JODM du 11 octobre $1902^{\text {lv }}$ donne les instructions à suivre pour la présentation des ressources de Madagascar en insistant sur la nécessité de faire connaître ces produits. Les administrateurs demandent ainsi l'envoi de cartes de chaque district consignant les concessions et leurs productions ${ }^{\mathrm{lvi}}$. Si le girofle s'expose, il est pour autant rarement décrit dans les catalogues d'exposition. L'essence, quant à elle, ne semble le plus souvent pas présentée dans les vitrines. Le grand public n'est en effet, pas ou peu, le débouché principal de l'essence et du clou de girofle, plutôt réservés aux industriels de la chimie, de l'alimentation, de la pharmaceutique et, dans une moindre mesure, de la parfumerie. 
Aussi, pour favoriser ces produits, l'Administration coloniale joue le rôle d'intermédiaire entre les industriels métropolitains et les exportateurs coloniaux. À titre d'exemple, le 14 février 1903 le JODM informe qu'une usine de distillation de girofle située à Courbevoie souhaite se rapprocher des producteurs de la Colonie pour assurer son approvisionnement de 30 tonnes de clous de girofle par mois ${ }^{\text {lvii }}$. De même, le 7 janvier 1922, au moment où la distillation se généralise au sein même de la Colonie, le JODM publie une offre de la part de la maison G.P Bertault qui sollicite, entre autres, des essences de girofle (clous, griffes ou feuilles) avec indication de la teneur en eugénollviii.

Ces débouchés multiples sont renforcés au milieu du siècle par l'engouement en Indonésie pour les cigarettes parfumées aux clous de girofle, les kreteks, dont Madagascar devient rapidement un fournisseur important (en complément de la production indonésienne) $)^{\text {lix }}$. Ils permettent notamment au secteur d'activité de se redresser après les difficultés de la Seconde Guerre mondiale (Danthu et alii, 2014). Ainsi, en 1951, des accords franco-indonésiens autorisent l'exportation de girofle entre Madagascar et l'Indonésie pour une valeur de 300 millions de francs ${ }^{1 \mathrm{x}}$. Ce système d'accords concerne l'Indonésie mais également la Pologne ${ }^{\mathrm{lxi}}$, la Tchécoslovaquie ${ }^{\mathrm{lxi}}$, l'Autriche $^{\text {lxii }}$ etc. Le JODM permet dès lors au gouvernement colonial de promouvoir ces accords et, ainsi, d'assurer un débouché aux producteurs locaux.

Des années 1960 à 1980, la 1 ${ }^{\mathrm{e}}$ République malgache pérennise les mesures prises par l'Administration coloniale en faveur du giroflier. À partir de 1992, Madagascar opère un virage politique en termes d'encadrement des filières de rente: la caisse de stabilisation des prix du girofle est supprimée et les mesures prises par l'Administration coloniale sont pour la plupart abandonnées. Cette « ignorance politique » (Dove, 1995) qui repose sur l'absence d'investissement étatique dans l'économie du pays et, en contrepartie, la suppression des différentes taxes, a pour conséquence la libéralisation complète de la filière.

\section{Conclusion}

Introduite à Madagascar, la culture du giroflier est adoptée rapidement par les colons et les Malgaches avant de devenir l'exclusivité de ces derniers. Requérant un "soin réduit » ${ }^{\text {kiv }}$ et offrant un double produit, le clou et l'essence, il colonise, entre 1896 et 1958, Sainte-Marie et la côte est de Madagascar et participe, tout au long de la période coloniale, à la croissance économique du pays.

Le rôle de l'Administration a été central dans l'encadrement de la filière comme dans la valorisation des produits du giroflier sur le marché national et international. Cette implication s'illustre par une double politique: assurer, en amont, une production quantitative et qualitative par l'accompagnement des cultivateurs et favoriser, en aval, les produits du giroflier auprès des acheteurs/consommateurs.

Cependant, l'expansion de cette culture est également à l'origine d'un bouleversement conséquent de la biodiversité malgache : le giroflier croît sur la côte est de la Grande-île et à Sainte-Marie au dépend d'autres cultures de rente ou vivrières (riz, café, culture maraîchère). De même, la distillation nécessite un volume conséquent en eaux et en bois et l'impact négatif de la production d'essence sur l'environnement a très tôt été 
signalé, en particulier concernant la consommation de bois-énergie qui favorise la déforestation.

Ces limites, associées à l'absence de contrôle de la filière qui autorise une concurrence déloyale exercée par certains exportateurs étrangers, sont aujourd'hui des éléments majeurs de la problématique giroflière à Madagascar. L'analyse de la trajectoire historique de la filière apporte dès lors des éléments de compréhension du contexte actuel qui permettraient de proposer des réponses adéquates afin d'en assurer l'avenir et la pérennité.

\section{BIBLIOGRAPHIE}

Bonneuil C., 1991, « Des savants pour l'Empire : les origines de l'ORSTOM », Cahiers pour l'histoire $d u$ CNRS, Éditions du CNRS, n¹0, 15 p.

Danthu P., Penot E., Ranoarisoa K.M, et alii, 2014, « The clove tree of Madagascar : a success story with an unpredictable future », Bois et forêts des tropiques, n³20 (2), pp. 83-96.

Danthu P., Razakamanarivo H., Deville-Danthu B. et alli, « The short and forgotten history of rubber in Madagascar: the first controversy between biodiversity conservation and natural resource exploitation", Bois et forêts des tropiques, n 328 (2), pp. 27-43.

Deleage C., Fremigacci J. (ss la dir.), 1990, Le bagnard et le girofle: un essai de mise en valeur par la main d'œuvre pénale coloniale, maîtrise en Histoire, Université Panthéon-Sorbonne, Paris, 133 p.

Deleigne M.C., 2010 « Les jardins scolaires des écoles du premier degré à Madagascar (1916-1951) », Histoire de l'éducation, n¹28, pp. 103-128.

Dove M.R., 1995, « Political versus techno-economic factors in the development of non-timber forest products: lessons from a comparison of natural and cultivated rubbers ", Southeast Asia and South America, Society and Natural Resources, n 8, pp.193-208.

Fremigacci J., 2014, État, économie et société coloniale à Madagascar : de la fin du XIXe siècle aux années 1940, Karthala, Paris, 616 p.

Levasseur S., Penot E. et Danthu P. (ss la dir.), 2012, Analyse des systèmes agricoles à base de girofliers à Sainte-Marie, Madagascar : entre héritage colonial et innovations paysannes, mémoire en vue de l'obtention du diplôme d'ingénieur agronome, Université d'Antananarivo-CIRAD, 74 p.

Martin P.J., 1991, «The Zanzibar clove industry», Economic Botany, vol. 45, n4, pp. 450-459.

Rahonintsoa E.T., 1978, Sainte-Marie de Madagascar : insularité et économie du girofle, Université de Madagascar, mémoire de maîtrise, Etablissement d'enseignement supérieur des lettres, 166 p.

Rasoa P., Rakotoarison J. (ss la dir.), 1994, Législation et réglementation sur la production et la commercialisation du girofle à Madagascar, maîtrise de droit, Université de Fianarantsoa, 85 p.

Zolesio J.P., « De Laire, Symrise, Historique », Cinquième Sens, 2016, http://olfatheque.com/ fiche-entreprise-1238-De-Laire.html, consulté le 13/02/2016. 
Toillier M., 2012, Rapport de recherches d'archives et bibliographiques : l'implantation de la culture du giroflier, Rapport de stage aux ANOM, CIRAD, Montpellier, $46 \mathrm{p}$.

Volper S., 2011, Du cacao à la vanille, Une histoire des plantes coloniales, éditions Quae, Versailles, 144 p.

\section{NOTES DE FIN}

i. Isnard H., 1951, « La colonisation agricole à Madagascar ", Revue de géographie alpine, Tome 39, n¹, pp. 97-125, p. 97.

ii. Hildebert Isnard (1904-1983) est un géographe et professeur, auteur de plusieurs ouvrages et articles consacrés à Madagascar dont : Madagascar, 1955, 1 volume, Paris, A. Colin, 215 p.

iii. Galliéni J.S, 1908, Neuf ans à Madagascar, Paris, Hachette, 371 p.

iv. Le Journal officiel de Madagascar et dépendances, organe de diffusion législatif, est consacré à la colonie. Il a été édité entre 1896 et 1958. Durant cette période, 628 périodiques consacrent un ou plusieurs articles au giroflier et à ses produits.

v. Decary R., août-septembre 1937, «Les débuts de la colonisation agricole à l'île SainteMarie de Madagascar ", Revue de botanique appliquée et d'agriculture coloniale, $\mathrm{n}^{\circ} 192-193$, pp. 610-618; Tourneur M., 1947, «Épices et aromates ", Encyclopédie de l'Empire français, Tome premier, Madagascar et Réunion, France, M. de Coppet (Éd.), pp. 310-321.

vi. Une quinzaine de rubrique «bulletin de territoire civils» et une dizaine de rubrique "renseignement économiques et commerciaux» concernant la province de Tamatave s'intéressent au girofle durant la période 1896-1903.

vii. Boiteau G., 1936, « Le girofle », Revue de Madagascar, vol. 38, n¹3, pp. 107-116.

viii. JODM, 12 octobre 1897, p. 5.

ix. JODM, 20 avril 1897, p. 5.

x. JODM, 19 février 1898, p. 7.

xi. JODM, 6 octobre 1898, p. 3.

xii. Galliéni J.S, 1908, Neuf ans à Madagascar, op.cit.

xiii. Émile Prudhomme est nommé, en 1896, inspecteur de l'Agriculture à Madagascar puis directeur en 1900, il devient directeur du jardin colonial de Nogent-sur-marne à partir de 1909.

xiv. Galliéni J.S, 1908, Neuf ans à Madagascar, op.cit.

xv. JODM, 4 février 1899, p. 4.

xvi. JODM, 13 septembre 1899, p. 5 .

xvii. Ibidem.

xviii. En 1913 et 1917, 7 et 9 immatriculations ont été publiées contre en moyenne 1 à 3 immatriculations par an sur la période 1896-1920.

xix. Selon Rahonintsoa, le premier atelier attesté reviendrait au colon Plaire en 1906 (Rahonintsoa E.T., 1978, Sainte-Marie de Madagascar : insularité et économie du girofle, Université de Madagascar, Etablissement d'enseignement supérieur des lettres, 332 p., p. 141). A. Ledreux cite quant à lui la date de 1911 (Ledreux A., juillet-août 1932, "Le giroflier à Sainte-Marie et à Madagascar ", L'agronomie coloniale, n¹75-176, pp. 1-22, p. 14). Nous estimons que la date de 1906 semble plus pertinente car Dussel indique que dès 1912, plus d'une tonne d'essence de girofle est déjà exporté de Madagascar (Dussel L., avril 1962, "Produits Malgaches », Bulletin de Madagascar, $\mathrm{n}^{\circ} 191$, pp. 325-338, p. 328.). De même le JODM indique dès 1902, l'investissement d'un colon pour la production d'essence de feuille de girofle (JODM, 19 novembre 1902, p. 17.). 
xx. Chiffres établis à partir des superficies fournies au sein des immatriculations de domaines dans le JODM entre 1896 et 1920.

xxi. Jean Gattefossé rapporte que la demande en eugénol a été considérable pendant la guerre pour les besoins de l'aviation. Cette essence est en effet employée comme plastifiant dans la fabrication des vernis transparents et des vernis colorés qui servaient à peindre les cocardes tricolores des ailes d'avions. Gattefossé J., octobre 1921, «Les végétaux aromatiques de Madagascar », Agronomie coloniale, n46, pp. 113-121, p. 113.

xxii. Otto M., 1909, "La vanilline synthétique », L'industrie des parfums d'après les théories de la chimie moderne, Paris, H. Dunod et E. Pinat, Éditeurs, pp. 400-419.

xxiii. Decary R., août-septembre 1937, « Les débuts de la colonisation agricole à l'île Sainte-Marie de Madagascar ", art.cit, p. 614.

xxiv. JODM, 19 novembre 1902, p. 17.

Xxv. Maistre J., 1955, « Le giroflier à Madagascar et Zanzibar », Agronomie tropicale, vol. $\mathrm{X}, \mathrm{n}^{\circ} 4, \mathrm{pp} .414-448$.

xxvi. JODM, 22 mai 1926, p. 14.

xxvii. JODM, 10 juillet 1926, p. 16.

xxviii. JODM, 7 août 1926, p. 11.

xxix. JODM, 10 novembre 1923, p.34

xxx. Maistre J., 1955, « Le giroflier à Madagascar et Zanzibar », art.cit.

xxxi. En 1929, le JODM rappelle à cinq reprises (23 février, 2 mars, 9 mars, 16 mars et 23 mars) l'obligation de déclarer leurs appareils sous peine de sanctions édictées dans l'article $2 \mathrm{du}$ décret du 23 novembre 1926. En effet, entre 1926 et 1929, une autorisation est publiée. Malgré ces rappels, seule une demande est déposée entre 1929 et 1938.

xxxii. JODM, 24 février 1897, p. 8.

xxxiii. JODM, 8 décembre 1898, p. 3.

xxxiv. JODM, 26 décembre 1936, p. 3.

xxxv. Secrétariat général du Gouvernement de Madagascar, Compte rendu officiel des travaux de la conférence économique réunie à Tananarive, Madagascar, Imprimerie officielle, 1919-1920, 622 p., p. 298.

xxxvi. JODM, 22 octobre 1921, p. 6.

xxxvii. Charles Edmond François (1887-1950) effectue une carrière de gouverneur des colonies. Il s'engage à Madagascar en 1920 comme agents de cultures. Il occupe successivement les postes de Directeur des jardins botaniques de Tananarive et Tamatave et d'inspecteur du service de la propagande et des stations d'essai avant de devenir inspecteur général chargé de la direction des services de l'agriculture.

xxxviii. François E., 1928, «La culture du giroflier à Madagascar ", Revue internationale de botanique appliquée et d'agriculture tropicale, n86, pp. 693-696, p. 695.

xxxix. JODM, 2 septembre 1933, p. 11.

xl. Témoignage de Dasy Armel, 73 ans, habitant du village d'Antetezam-Pafana, recueilli en avril 2015 par Mathilde Cocoual.

xli. Anonyme, 1909, "Fonctionnement des stations d'essais pendant l'année 1908 ", Bulletin économique de Madagascar, pp. 181-265, p. 191.

xlii. Anonyme, 1907, "Les essais de culture dans les stations agricoles de Marovoay et de l'Ivoloina en 1907 », Bulletin économique de Madagascar, pp. 361-370, p. 369.

xliii. JODM, 27 avril 1897, p. 8 .

xliv. JODM, 25 décembre 1901, p. 2.

xlv. JODM, 20 décembre 1902, p. 46.

xlvi. JODM, $1^{\mathrm{e}}$ juillet 1922, p. 5 .

xlvii. JODM, 27 avril 1897, art.cit, p. 8 
xlviii. JODM, 10 décembre 1898, p. 2.

xlix. JODM, 15 novembre 1930, p. 7.

1. JODM, 9 septembre 1922, p. 3.

li. JODM, 19 octobre 1957, p. 14.

lii. Dasy Armel rapporte que, durant les années 1960-70, le prix du clou, permettait de subvenir aux besoins des familles et d'acheter les produits de première nécessité.

liii. JODM, 16 mars 1899, p. 6.

liv. JODM, 30 octobre 1901, p. 2.

lv. JODM, 11 octobre 1902, p. 3.

lvi. Ibidem.

lvii. JODM, 14 février 1903, p. 4.

lviii. JODM, 7 janvier 1922, p. 29.

lix. François E., 1936, "girofle et giroflier », Revue de botanique appliquée et d'agriculture coloniale, $n^{\circ} 180$, p. 589-608, p.591.

lx. JODM, 29 septembre 1951, p. 21.

lxi. JODM, 29 mai 1948, p. 15.

lxii. JODM, 23 mai 1953, p. 59.

lxiii. JODM, 23 janvier 1954, p. 41.

lxiv. Le giroflier croît assez facilement mais nécessite néanmoins un suivi régulier, certes moins important que d'autres denrées coloniales telles la vanille ou le café.

\section{RÉSUMÉS}

Madagascar est aujourd'hui le second producteur mondial après l'Indonésie et premier exportateur mondial de clous et d'essence de girofle.

Le giroflier a été introduit à Madagascar il y environ deux siècles mais le contexte de son introduction et de son expansion a été peu étudié et de nombreuses questions relatives aux conditions de production et de commercialisation durant la période coloniale et postcoloniale restent encore en suspens.

En croisant les données recueillies dans le Journal officiel de Madagascar et Dépendances, publié entre 1896 et 1958, et les écrits d'ingénieurs agronomes et d'administrateurs coloniaux, l'objectif de cet article est de dresser un tableau géographique et historique de l'évolution de la filière du giroflier à Madagascar.

Géographique tout d'abord en esquissant les modalités d'expansion des plantations qui, si elles semblent au premier abord s'étendre de manière graduelle depuis l'île de Sainte-Marie vers la côte est de Madagascar, répondent en réalité à une implantation au "coup par coup » dont les modalités sont liées à des facteurs physiques (sol, climat, proximité de voies de communication fluviales ou terrestres) mais aussi et surtout à des conditions matérielles et humaines spécifiques. L'étude des sources recueillies permet ainsi d'esquisser l'évolution géographique de ces implantations durant la première moitié $\mathrm{du} \mathrm{XX}^{\mathrm{e}}$ siècle et l'essor des ateliers de distillation des feuilles et griffes de girofle qui se multiplient à partir des années 1920.

Historique, ensuite, par l'étude de l'action de l'Administration coloniale pour favoriser et encadrer une production qui, avec les autres cultures dites « riches » (café, cacao, vanille, plantes à parfum) doit permettre à la colonie d'établir une balance commerciale équilibrée. Sur fond de lutte impériale avec les productions britanniques des îles de Zanzibar et de Pemba, alors premier producteur mondial de clous de girofle au début du siècle, l'Administration encourage les colons mais également les Malgaches à adopter cette culture. Le giroflier devient dès lors un symbole incontournable de l'île de Sainte-Marie puis de la côte est de la Grande-Île. En parallèle, les administrateurs mènent une politique active de publicité auprès des consommateurs 
métropolitains tout en promouvant la recherche de débouchés transversaux: production d'eugénol pour la pharmacologie, mise en relation producteurs et importateurs, accords commerciaux avec des pays étrangers, etc.

En privilégiant cette approche historico-géographique, cette article espère apporter des clés de lecture permettant de comprendre l'adoption et le développement de cette culture à Madagascar, d'apporter des éléments de compréhension de son évolution actuelle et, par la même occasion d'en envisager l'avenir.

Madagascar, the world's second largest producer of cloves, has experienced a drop in production because of the ageing of its productive tree stock. The reduced productivity of this resource has prompted a joint study and broad reflection about its future and its past origins. Initiated by the International Development and Agronomic Research Center (CIRAD) and the local universities of Madagascar, this multidisciplinary research should help the local clove industry better understand changes in the international market critical to Madagascar's clove exports and foreign exchange earnings.

The introduction of non-endemic clove trees to Madagascar and the expansion of their production have not previously been widely studied and several key questions regarding the conditions of production and the marketing sales strategy during both the colonial and postcolonial periods remain unanswered. By looking at data collected from the Journal officiel de Madagascar et Depandances, published between 1896 and 1958, and written statements by agronomists and colonial civil servants (eg. Raymond Decary, Emile Prudhomme and Edmond Francois) this paper lays out a geographical and historical perspective of the evolution of the clove industry in Madagascar.

First, the geographic evolution is outlined. Based on a review of the source data and literature, there was a gradual expansion of clove plantations from the area l'Ile de Sainte Marie towards the eastern coast of Madagascar during the first half of the $20^{\text {th }}$ century. The expansion occurred in small, unplanned steps influenced by a combination of physical factors (soil, climate, and proximity to river or overland transport) and by specific entrepreneurial and marketability factors (access to finance until the first harvests, availability of farm labor, and good physical infrastructure to enable exports). Beginning in about the 1920s, small-scale clove distilleries first appeared and grew in a scattered fashion around the coastal areas.

The historical evolution of clove production can be studied through papers written during the colonial period. French colonial administrators in Madagascar encouraged the production of marketable, export-earning crops, known as "rich crops", such as cloves, coffee, chocolate, vanilla, and plants for the perfume industry. The objective was to enable the colony to have a favorable trade balance. The strategy was also part of a colonial rivalry with the British colonies producing similar crops in the islands of Zanzibar and Pemba located off the coast of Tanzania. At that time (1900), Tanzania was the largest world producer of cloves and the French colonial administration wanted to encourage French colonial and local Madagascar farmers to introduce this crop which was rapidly to become a defining symbol of l'Ile de Ste Marie and the Eastern Coast. In parallel, the colonial administration put producers in direct contact with importers and prepared commercial agreements. It also spearheaded an active marketing campaign in French cities to incorporate cloves into daily consumption habits and worked to identify opportunities for using clove extract (eugenol) in the pharmaceutical industry.

By using an historical and geographic approach, this paper tries to understand the past development and growth of this industry and garner insights into its future prospects. 
INDEX

Mots-clés : giroflier, clou de girofle, essence de girofle, Madagascar, colonisation agricole, histoire

Keywords : clove tree, cloves, essential oil, Madagascar, colonization, agriculture, historical

\section{AUTEURS}

\section{PASCAL DANTHU}

Université Côte d'Azur, Centre de la Méditerranée Moderne et Contemporaine, 98 Boulevard Edouard Herriot, 06200 Nice Cedex 2, France (adresse actuelle : 610 Route de Tourrettes, 83440 Tourrettes, France); m.cocoual@yahoo.fr Centre de Coopération Internationale en Recherche Agronomique pour le Développement (CIRAD), UPR HortSys, Université de Montpellier, Campus de Lavalette, 34398 Montpellier cedex 5, France ; pascal.danthu@cirad.fr 